Revue internationale P.M.E.

Économie et gestion de la petite et moyenne entreprise

\title{
Qualité et sous-traitance dans l'industrie italienne : quelques résultats d'une étude empirique
}

\section{Emilio Esposito et Mario Raffa}

Volume 5, numéro 2, 1992

URI : https://id.erudit.org/iderudit/1008139ar

DOI : https://doi.org/10.7202/1008139ar

Aller au sommaire du numéro

Éditeur(s)

Presses de l’Université du Québec

ISSN

0776-5436 (imprimé)

1918-9699 (numérique)

Découvrir la revue

Citer cet article

Esposito, E. \& Raffa, M. (1992). Qualité et sous-traitance dans l'industrie italienne : quelques résultats d'une étude empirique. Revue internationale P.M.E., 5(2), 57-82. https://doi.org/10.7202/1008139ar

\section{Résumé de l'article}

À partir de quelques évidences empiriques, ce travail propose une contribution au débat sur la nature des nouveaux rapports liant les grandes entreprises de haute technologie et les petites entreprises de sous-traitance. Le cadre de référence spécifiquement considéré est l'industrie italienne. L’approche utilisée étudie la façon dont la grande entreprise de haute technologie sollicite le développement des petites entreprises de sous-traitance. L'analyse a été menée à partir d'un modèle de transmission de la technologie entre les grandes et les petites entreprises. Dans le modèle, la technologie est décrite comme un ensemble de quatre composantes : machines, capacités professionnelles, règles d'organisation et documents. Les vecteurs de liaison transportant ces composantes entre les entreprises se définissent comme canaux de transmission de la technologie.

L'enquête repère donc ces canaux de transmission de la technologie entre la grande entreprise de haute technologie et les petites entreprises de soustraitance. La conclusion à laquelle conduit cette recherche est que la grande entreprise de haute technologie, afin de gérer le cycle de production dans le respect de standards de qualité élevés, sollicite et soutient la croissance des petites entreprises de sous-traitance. La petite entreprise abandonne l'optique de court terme et s'oriente vers un processus de croissance quantitatif et qualitatif.
Ce document est protégé par la loi sur le droit d'auteur. L'utilisation des services d'Érudit (y compris la reproduction) est assujettie à sa politique d'utilisation que vous pouvez consulter en ligne.

https://apropos.erudit.org/fr/usagers/politique-dutilisation/ 


\title{
Qualité et sous-traitance dans l'industrie italienne : quelques résultats d'une étude empirique*
}

\author{
Emilio ESPOSITO \\ Mario RAFFA** \\ ODISSEO-DIS, Université de Naples
}

\begin{abstract}
RÉSUMÉ
A partir de quelques évidences empiriques, ce travail propose une contribution au débat sur la nature des nouveaux rapports liant les grandes entreprises de haute technologie et les petites entreprises de sous-traitance. Le cadre de référence spécifiquement considéré est l'industrie italienne. L'approche utilisée étudie la façon dont la grande entreprise de haute technologie sollicite le développement des petites entreprises de sous-traitance. L'analyse a été menée à partir d'un modèle de transmission de la technologie entre les grandes et les petites entreprises. Dans le modèle, la technologie est décrite comme un ensemble de quatre composantes: machines, capacités professionnelles, règles d'organisation et documents. Les vecteurs de liaison transportant ces composantes entre les entreprises se définissent comme canaux de transmission de la technologie.

L'enquête repère donc ces canaux de transmission de la technologie entre la grande entreprise de haute technologie et les petites entreprises de soustraitance. La conclusion à laquelle conduit cette recherche est que la grande entreprise de haute technologie, afin de gérer le cycle de production dans le
\end{abstract}

* Ce travail illustre les résultats empiriques d'une recherche qui met en évidence le rôle et la spécificité de la petite entreprise italienne sous-traitante de grandes entreprises high-tech. La recherche a bénéficié du financement du C.N.R. - Institut de recherches sur les activités tertiaires (n. 8905320. CT 13). Les aspects théoriques du modèle proposé par les auteurs ont été présentés au 36th ICBS World Conference qui a eu lieu à Vienne du 24 au 26 juin 1991 et où il a reçu le prix de d'Excellence.

** E. Esposito et M. Raffa sont respectivement chercheur et professeur associé à la Facoltà di Ingegneria de l'Université de Naples. Ils sont tous deux chercheurs de ODISSEO, Observatoire sur l'organisation et l'innovation technologique du Département d'informatique et systémique, Université de Naples. Adresse: ODISSEODIS, Via Diocleziano 328, 80124 Napoli (Italia) 
respect de standards de qualité élevés, sollicite et soutient la croissance des petites entreprises de sous-traitance. La petite entreprise abandonne l'optique de court terme et s'oriente vers un processus de croissance quantitatif et qualitatif.

\section{ABSTRACT}

Starting with some empirical evidences, this paper aims at contributing to the debate on the new relationships between high tech firms and small subsuppliers. The prevaling framework is the Italian industry. The approach investigates how large high tech firms stimulate the development of small subsuppliers. The analysis is carried out by making reference to a model of technology transfer between large and small firms. In the model, technology is viewed as a set of four components : machinery, professional skills, organizational rules and documents. These components are transported between firms through linkage vectors refered to as technology transfer channels.

The survey thus identifies technology transfer channels between high tech firms and small suppliers. The conclusion drawn by this research is that large high tech firms, in order to manage the production cycle and simultaneously me日t high quality standards, stimulate and support the growth of the small subsuppliers. Small firms drop the short term perspective and tend to a quantity and quality growth process.

\section{RESUMEN}

Este trabajo se propone, a partir de algunas evidencias empiricas, el objetivo de dar una contribucion al debate sobre la natureza de las nuevas relaciones entre la gran empresa High tech y las pequenas empresas sub abastecedoras con particular referencia a la industria italiana. El estudio realizado ve como la gran empresa High tech solicita el desarrollo de las pequenas empresas sub abastecedoras. El analisis viene conducida sobre la base de un modelo de transmision de la tecnologia entre grandes y pequenas empresas. En el modelo, la tecnologia viene vista como un conjunto de cuatro componentes (maquinas, profesionalidad, reglas organizativas y documentos). Las lineas de enlace entre la empresa sobre las cuales vienen transportados estos componentes son definidos canales de transmision de la tecnologia.

A traves de la indagacion se individuan los canales de transmision de la tecnologia entre la gran empresa High tech y las pequenas empresas sub abastecedoras. La conclusion a la cual llega este estudio es que la gran empresa High tech, para gestionar el ciclo productivo en respecto de los elevados standard de calidad, solicita y sostiene el crecimiento de las pequenas empresas sub abastecedoras. La pequena empresa abandona la optica de breve periodo y se orienta hacia un proceso de crecimiento tanto cuantitativo como cualitativo. 


\section{Introduction'}

À partir des années 1980, de nombreuses recherches ont montré qu'une rapide évolution des rapports de sous-traitance est en cours, tant sur le plan national qu'international (Del Monte, 1991).

Les différentes enquêtes soulignent que les rapports entre petites et grandes entreprises, et en particulier le rapport de sous-traitance, ne peuvent plus être ramenés à la simple recherche, de la part de la grande entreprise, de coûts externes plus bas. Ils dépendent également d'un arbitrage entre des facteurs techniques (contrôle des coûts, système de qualité, fiabilité des livraisons), financiers (le sous-traitant doit avoir une structure financière solide) ainsi que des facteurs plus généraux (capacité de participer au développement de nouveaux produits).

En particulier, la grande entreprise de haute technologie, qui réalise un produit dont la complexité est le résultat de la qualité de chacun des composants et de la complexité de leur intégration, a besoin de se lier à des sous-traitants capables de réaliser les parties et les composants en respectant les standards de qualité élevés indispensables à la réalisation du produit. Pour ce faire, la petite entreprise doit s'équiper de technologies cohérentes, c'est-à-dire compatibles avec le système technologique du commettant.

La grande entreprise de haute technologie devient ainsi partie intégrante d'un système où convergent des expériences et des connaissances qui ne sont pas seulement intérieures à l'entreprise elle-même, mais qui se projettent à l'extérieur jusqu'aux petites entreprises sous-traitantes. Dans cette perspective, la grande entreprise, afin d'améliorer sa position compétitive, doit optimiser aussi bien les ressources disponibles à l'intérieur que les composantes extérieures, c'est-à-dire qu'elle doit guider le développement des petites entreprises qui exécutent une partie de la production.

Partant d'un modèle interprétatif des modalités de transmission de la technologie entre les entreprises, ce travail enquête sur la nature des nouveaux rapports qui lient les grandes entreprises de haute technologie et les petites entreprises sous-traitantes (Esposito et Raffa, 1991).

À partir d'un échantillon constitué de 58 entreprises localisées en Italie et sous-traitantes de grandes entreprises de haute technologie italiennes (Aeritalia,

1. Bien que le travail ait été effectué en collaboration par les deux auteurs, les parties 2, 4.1, 4.2 peuvent être attribués plus particulièrement à E. Esposito et les parties 3, 4.3, 4.4 à M. Raffa. 
Alfa Avio, Fiat Auto, Magnaghi, Olivetti, Selenia, etc.) $)^{2}$ du secteur électronique, aéronautique, automobile ou de composants électroniques divers, on définit et on construit un modèle explicatif des modalités de transmission de la technologie entre les entreprises en général, et entre les grandes entreprises de haute technologie et les petites entreprises sous-traitantes en particulier. Pour étudier les petites entreprises, les chercheurs d'ODISSEO ont interviewé directement les différents responsables, en se basant sur un questionnaire semi-ouvert spécifique. L'échantillon utilisé pour cette étude est composé d'entreprises sous observation depuis le début de 1988.

Dans le modèle, on relève onze canaux de transmission de la technologie perçue comme un ensemble de quatre composantes : règles d'organisation, machines, documents et capacités professionnelles. On a également donné un poids à la contribution de chacun des canaux, considérés comme autant de vecteurs de transmission de la technologie, et on met en évidence le rôle qu'ont les vecteurs qui servent de support à la qualité dans la définition du nouveau rapport entre les petites entreprises sous-traitantes et les grandes entreprises de haute technologie.

Les résultats de la recherche mettent en évidence une tendance homogène et nationale : les grandes entreprises choisies sont représentatives de l'industrie de haute technologie italienne, alors que les petites entreprises étudiées représentent les entreprises les plus dynamiques, c'est-à-dire celles qui ont réussi à suivre durant ces dernières années les grandes entreprises de haute technologie (Pratten, 1991 ; Lassini et Mariti, 1991). Naturellement, il y a le reste des petites entreprises qui, tout en étant en rapport avec la grande, ne réussissent pas à suivre les standards que celle-ci impose. Ceci engendre une véritable articulation des rapports entre les grandes entreprises de haute technologie et les petites entreprises sous-traitantes : à côté des petites entreprises qui réussissent à jouer un rôle actif, d'autres, à des niveaux différents, sont reléguées à des rôles marginaux. La littérature met en évidence comment les phénomènes du même type se produisent dans d'autres pays européens (Imrie et Morris, 1988 ; Lamming, 1986 ; Sako, 1987). De récentes études ont mis en évidence la façon dont, durant ces dernières années, les barrières technologiques et organisationnelles qui s'élevaient entre les entreprises se sont abaissées (Lazerson, 1990) et le fait que sur le plan international, une véritable évolution de la structure industrielle des différents pays est en cours (Auerbach, 1988).

2. Depuis 1991, Aeritalia et Selenia ont fusionné, donnant naissance à Alenia. 


\section{La qualité comme facteur qui influence les nouveaux rapports}

Dans les années 1980, la qualité est devenue un facteur stratégique pour le succès de l'entreprise, le carrefour par où passent marché, développement de projets, production et vente. Selon certaines approches (Ishikawa, 1982), la mission elle-même de l'entreprise est la qualité, le profit en étant une conséquence. La qualité devient un moyen de gouverner l'entreprise dans la mesure où un quelconque résultat d'un point de vue qualitatif ne peut être atteint que si toutes les fonctions concernées par le processus tendent à poursuivre l'objectif final (Juran, 1989 ; Deming, 1982 ; Crosby, 1979). Pour certains auteurs, le concept de qualité inclut aussi la satisfaction du client (Ishikawa, 1982), lequel devient partie intégrante et indispensable du système de qualité, donc un élément clé du succès de l'organisation. En outre, le fournisseur (donc également le soustraitant) n'est plus considéré comme un élément extérieur duquel il faut obtenir les produits et les services au prix le plus bas possible ; il est considéré comme un élément du système, dont la grande entreprise fait également partie, avec lequel il faut atteindre le meilleur résultat possible, à la plus grande satisfaction possible du système tout entier. Par conséquent, de nouveaux rapports entre fournisseurs, sous-traitants et commettants se développent. C'est ainsi que l'approche compétitive de Porter est remise en question (Hax et Majluf, 1991).

Tout ceci modifie de façon significative le rapport entre petites et grandes entreprises (secteur de haute technologie en particulier) où les problèmes liés à la qualité sont très pressants (Fenneteau, 1990). Le rapport avec le sous-traitant ne se fonde plus exclusivement sur le prix, mais sur un arbitrage entre prix, analyse financière, capacité technologique, capacité de réponse et capacité de développer des projets. De cette façon, on pousse le sous-traitant à devenir partie intégrante de l'entreprise (par exemple, à travers des contrats de longue durée) et, dans certains cas, à travers différentes formes et degrés d'accès à une partie intérieure de l'entreprise (par exemple, libre accès à la salle de conception afin de mieux intégrer les différentes activités). Ce nouveau rapport implique de grands changements, que ce soit dans la grande entreprise de haute technologie ou dans la petite entreprise sous-traitante.

Les problèmes liés à la qualité, prioritaires pour la grande entreprise de haute technologie, intéressent toujours davantage le système entier des entreprises. On assiste ainsi à un changement graduel des rapports entre les entreprises, en particulier ceux liant les grandes et les petites entreprises. Les petites entreprises abandonnent l'optique de court terme qui se révèle incapable de suivre les changements rapides qu'impose le nouveau rapport qui s'instaure avec la grande entreprise de haute technologie. Ce rapport représente également un défi pour la petite entreprise, car il est plus complexe (dans la mesure où il 
impose de profondes modifications visant la culture, la technologie et l'organisation) et il les implique davantage (puisque la petite entreprise devient un acteur et n'est plus un sujet, souvent passif, du processus de production). Pour la petite entreprise, ce changement sur le plan de l'organisation, de la culture et de la technologie est une nécessité, puisque la petite entreprise orientée dans une optique de court terme renonce dès le départ à un rôle moteur que seule la croissance technologique peut lui garantir.

Pour la grande entreprise, ce changement est nécessaire afin d'éviter deux conséquences négatives : rester prisonnière d'un environnement qui, non sollicité dans une optique de croissance, n'est pas en mesure de répondre à la demande de qualité des marchés ; risquer d'être abandonnée par les petites entreprises les plus dynamiques qui renoncent à acquérir des commandes qui ne présentent pas un niveau qualitatif suffisant. Dans cette optique, la grande entreprise doit se donner pour objectif de solliciter et de soutenir la croissance des petites entreprises sous-traitantes.

\section{Les entreprises de l'échantillon}

Les résultats de la recherche se fondent sur un échantillon de 58 petites entreprises italiennes sous-traitantes de grandes entreprises de haute technologie italiennes. La subdivision des entreprises par classes en fonction du nombre de salariés montre que $29 \%$ des entreprises comptent moins de 20 salariés, alors que $43 \%$ appartiennent à la classe qui va de 21 à 50 salariés, tandis que $10 \%$ occupent la classe qui va de 51 à 100 salariés et $17 \%$ ont plus de 100 salariés (tableau 1).

TABLEAU 1

Distribution des entreprises par classes de salariés

\begin{tabular}{lcc}
\hline Classes de salariés & Nombre d'entreprises & \% d'entreprises \\
\hline Jusqu'à 20 & 17 & 29,3 \\
De 21 à 50 & 25 & 43,1 \\
De 51 à 100 & 6 & 10,3 \\
Plus de 100 & 10 & 17,3 \\
\hline Total & $\mathbf{5 8}$ & $\mathbf{1 0 0 , 0}$ \\
\hline
\end{tabular}

Source : ODISSEO-DIS, Facoltà di Ingegneria, Naples

Les 58 entreprises étudiées réalisent des parties, des composants et des produits finis pour des grandes entreprises qui opèrent dans des secteurs 
d'activité profondément différents entre eux, mais qui ont en commun la réalisation de produits devant se conformer à des normes de qualité, de fiabilité et de sécurité très serrées.

Dans le tableau 2, on voit qu'Aeritalia, Selenia et Fiat Auto impliquent dans leur cycle de production 21 entreprises ; 12 réalisent des fournitures en sous-traitance pour Ansaldo et Magnaghi ; 10 travaillent pour Olivetti ; Alfa Avio est client de 9 entreprises ; 4 entreprises comptent parmi leurs commettants Fiat Aviazione et IBM.

TABleAU 2

Les principaux commettants des petites entreprises sous-traitantes

\begin{tabular}{|c|c|c|c|c|c|}
\hline $\begin{array}{l}\text { Principaux } \\
\text { commettants }\end{array}$ & $1-20$ & \multicolumn{2}{|c|}{ Classes de salariés } & $>100$ & $\begin{array}{l}\text { Total des } \\
\text { entreprises }\end{array}$ \\
\hline Selenia & 7 & 10 & 3 & 1 & 21 \\
\hline Aeritalia & 6 & 10 & 2 & 3 & 21 \\
\hline Fiat Auto & 3 & 7 & 4 & 7 & 21 \\
\hline Magnaghi & 5 & 7 & 0 & 0 & 12 \\
\hline Ansaldo & 5 & 4 & 2 & 1 & 12 \\
\hline Olivetti & 4 & 1 & 4 & 1 & 10 \\
\hline Alfa Avio & 2 & 6 & 0 & 1 & 9 \\
\hline Fiat Aviazione & 0 & 4 & 0 & 0 & 4 \\
\hline IBM & 1 & 1 & 0 & 2 & 4 \\
\hline Autres & 12 & 13 & 4 & 4 & 33 \\
\hline
\end{tabular}

Source : ODISSEO-DIS, Facoltà di Ingegneria, Naples

Le tableau 3 montre la dimension des entreprises selon l'année de naissance. Seize entreprises ont plus de 25 ans, dix ont commencé leur activité entre 1966 et 1970 ; vingt sont nées entre 1971 et 1975 ; sept entre 1976 et 1980, et à peine cinq entreprises sont nées après 1980 . À l'exception de quelques microentreprises, peu nombreuses, les grandes entreprises de haute technologie impliquent dans leur cycle de production des entreprises qui ont au moins dix ans d'expérience et d'activité dans leurs secteurs d'appartenance respectifs.

Les petites entreprises de l'échantillon mettent en œuvre une politique limitée de diversification des clients. De fait, chaque entreprise développe des activités pour un noyau restreint de grandes entreprises (en moyenne 2,5 grandes entreprises pour chaque petite entreprise), ce qui permet d'obtenir l'avantage de l'intégration avec les grands commettants et, en même temps, de ne pas perdre les avantages liés à la diversification. En effet, en évitant de rester lié à un unique commettant, on réussit mieux à surmonter d'éventuelles crises dans les 
rapports avec un commettant donné et les éventuelles tendances négatives du marché de chaque commettant qui, comme chacun sait, se répercutent sur les commandes du sous-traitant.

Les entreprises étudiées couvrent un champ assez vaste d'activités productives. On a pu distinguer au moins 10 secteurs différents : équipement, charpenterie moyenne et légère et travail des tôles, mécanique de précision, usinage de composants en alliage léger, construction de composants en matériaux composites, tôlerie pour l'électronique et pour les cadres électriques, câblages et montages électriques, construction d'appareillages électriques et électroniques, produits de poudre de métal et travail des matériaux en plastique.

TABleaU 3

Distribution des entreprises par classes de salariés et par année de naissance

\begin{tabular}{lccccc}
\hline Année de & \multicolumn{5}{c}{ Classes de salariés } \\
nalssance & $\mathbf{1 - 2 0}$ & $\mathbf{2 1 - 5 0}$ & $\mathbf{5 1 - 1 0 0}$ & $\mathbf{> 1 0 0}$ & $\begin{array}{c}\text { Total des } \\
\text { entreprises }\end{array}$ \\
\hline Avant 1965 & 1 & 10 & 1 & 4 & 16 \\
$1966-1970$ & 4 & 4 & 1 & 1 & 10 \\
$1971-1975$ & 7 & 8 & 3 & 2 & 20 \\
$1976-1980$ & 2 & 3 & 1 & 1 & 7 \\
$1981-1985$ & 1 & 0 & 0 & 0 & 1 \\
Après 1985 & 2 & 0 & 0 & 2 & 4 \\
\hline Total & $\mathbf{1 7}$ & $\mathbf{2 5}$ & $\mathbf{6}$ & $\mathbf{1 0}$ & $\mathbf{5 8}$ \\
\hline
\end{tabular}

Source : ODISSEO-DIS, Facoltà di Ingegneria, Naples

La plus grande partie des entreprises travaille dans le secteur de la mécanique de précision (environ $48 \%$ ). Viennent ensuite les secteurs de l'usinage de composants en alliages légers (environ $19 \%$ ) et de la charpenterie moyenne/ légère (environ $17 \%$ ). Quatre entreprises (environ $7 \%$ ) travaillent dans l'industrie des matières plastiques, trois $(6 \%)$ dans la construction d'appareillages électriques et électroniques et trois autres dans le secteur des produits de poudre de métal. Une seule entreprise de l'échantillon travaille dans le secteur des équipements aéronautiques (ce qui s'explique par les nombreuses barrières technologiques et de marché que rencontrent les petites entreprises qui ne sont pas nées dans ce secteur). Une seule entreprise exerce aussi son activité dans le secteur des matériaux composites, une technologie qui ne s'est développée que récemment. Aussi, puisque la ressource humaine joue un rôle fondamental dans le travail de tels matériaux, l'acquisition du savoir-faire requiert de longues périodes de formation. 


\section{Le modèle de référence}

Les canaux à travers lesquels la grande entreprise influence le développement des petites entreprises peuvent être étudiés et analysés en considérant le flux de technologie qui circule depuis la grande jusqu'à la petite entreprise. Les canaux peuvent être considérés comme de véritables vecteurs de liaison entre deux entreprises transportant les différentes composantes de la technologie. Le poids des éléments de la technologie transmis par les vecteurs peuvent être évalués en utilisant le modèle de circulation de la technologie indiqué dans la figure 1 (page suivante).

La technologie de la grande entreprise ou de la petite entreprise est considérée comme étant l'ensemble des connaissances englobées dans le matériel informatique ou autre, dans les logiciels et dans les ressources humaines (The Technology Atlas Team, 1987).

Dans cette acception, par technologie, on entend une application systémique de différentes composantes : machines, capacités professionnelles, documents, règles d'organisation. Par machines, on entend toutes les technologies incorporées dans les objets, les composants et les parties, les instruments et les systèmes ; par capacités professionnelles, les connaissances détenues par les personnes, c'est-à-dire l'ensemble de l'habileté des ressources humaines ; par documents, les technologies incorporées sous forme d'idées et d'informations dans des manuels, des articles, des mémoires et autre documentation; et enfin, par règles d'organisation, nous visons les technologies incorporées sous forme de procédures et de liens d'organisation. Toutes ces composantes sont étroitement liées entre elles, et c'est pourquoi elles peuvent être considérées comme les nœuds d'un système d'où partent et où arrivent des sollicitations provenant aussi bien des autres composantes que de l'environnement extérieur.

En utilisant le modèle proposé, il a été possible de repérer les principaux canaux de transmission de la technologie : flux de matériaux à l'état brut ; transmission de parties et composants semi-finis ; appareillages pour des productions spécifiques; assistance à travers des activités de formation entreprises par le client sur des sujets particuliers (qualité, méthodologies de production, machines spécifiques) ; visites et suggestions du commettant ; collaboration de début de commande (où en quelque sorte on transmet la philosophie du programme); documents écrits, projets et autres flux d'information sous forme de papier ; réunion dans les locaux du commettant ; visites du client ayant pour but de vérifier l'avancement du programme et les conditions générales de santé du sous-traitant ; suggestions du client pour l'adoption du contrôle qualité (CQ) et intervention directe du client pour améliorer la fonction CQ. 
FIGURE 1

La diffusion de la technologie depuis la grande entreprise jusqu’à la petite entreprise à travers les onze canaux de transmission

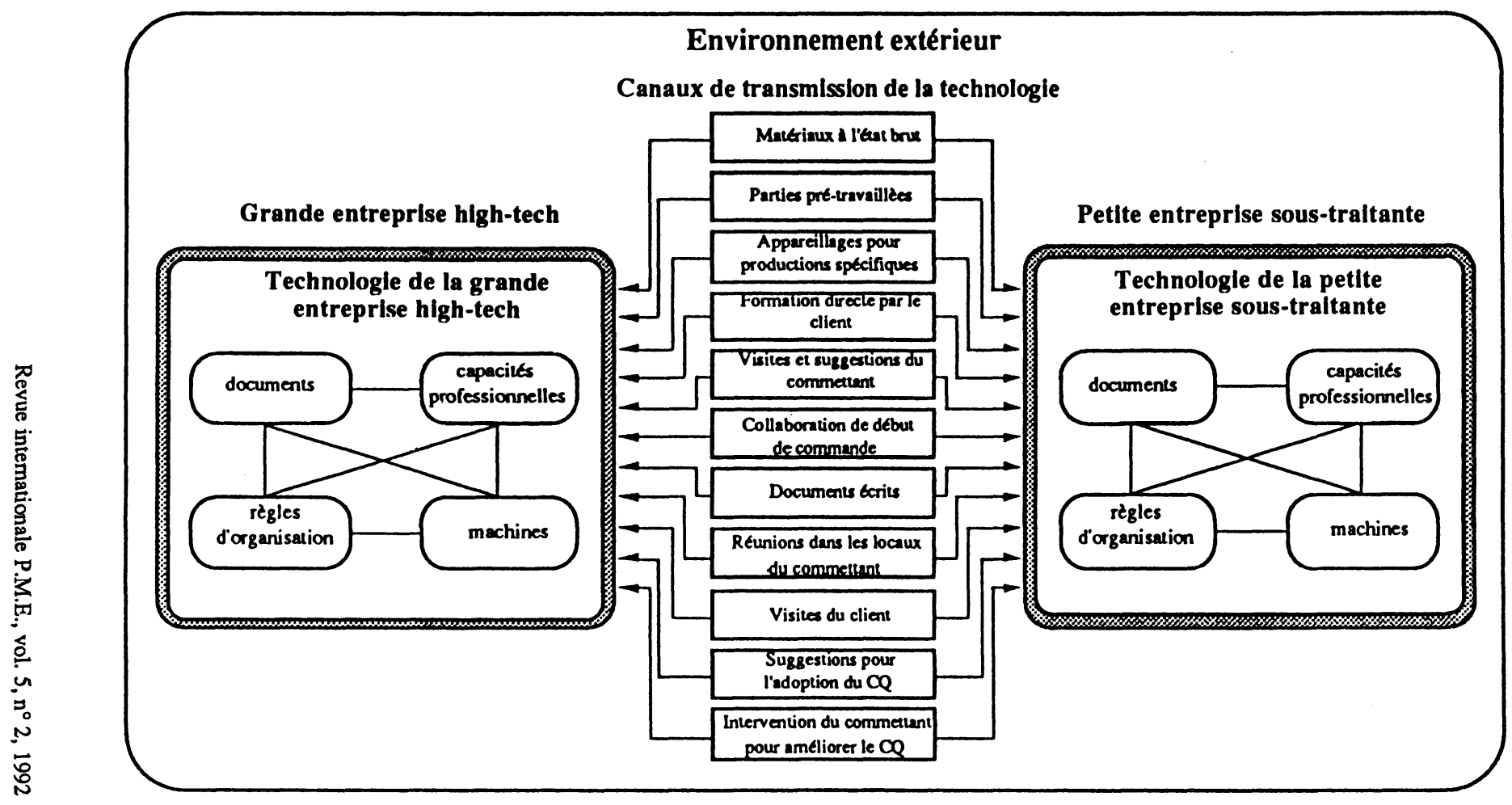


La croissance des petites entreprises sous-traitantes dépend de la fréquence et de l'intensité de la technologie qui circule entre la grande et la petite entreprise. Elle est induite par les grandes entreprises à travers tous les canaux de diffusion et concerne simultanément les quatre composantes de la technologie.

Dans les tableaux 4 et 5 sont reportés en valeur absolue et en pourcentage les poids des canaux de diffusion de la technologie. Le principal canal de transmission de la technologie est celui des documents écrits, avec un pourcentage d'environ $95 \%$. Deux autres canaux très utilisés sont ceux des réunions dans les locaux du commettant et la collaboration au début des commandes (79\% des entreprises interviewées). Les visites du commettant au sous-traitant pour suivre et contrôler l'avancement du programme et les suggestions du client afin de faire adopter le système du contrôle de qualité (67\% des cas) sont également deux autres canaux importants.

TABLEAU 4

Le poids des canaux de transmission de la technologie depuis la grande entreprise de haute technologie jusqu'à la petite sous-traitante

(valeur absolue)

\begin{tabular}{|c|c|c|c|c|c|}
\hline \multirow{2}{*}{$\begin{array}{l}\text { Canaux de transmission } \\
\text { de la technologie }\end{array}$} & \multicolumn{4}{|c|}{ Classes de salariés } & \multirow[t]{2}{*}{ Total } \\
\hline & $1-20$ & 21-50 & $51-100$ & $>100$ & \\
\hline Documents écrits & 15 & 25 & 6 & 9 & 55 \\
\hline $\begin{array}{l}\text { Collaboration de début } \\
\text { de commande }\end{array}$ & 11 & 22 & 5 & 8 & 46 \\
\hline $\begin{array}{l}\text { Réunions dans les locaux } \\
\text { du commettant }\end{array}$ & 13 & 19 & 6 & 8 & 46 \\
\hline Visites du client & 10 & 20 & 5 & 8 & 43 \\
\hline $\begin{array}{l}\text { Suggestions pour l'adoption } \\
\text { du CQ }\end{array}$ & 8 & 20 & 5 & 6 & 39 \\
\hline $\begin{array}{l}\text { Visites et suggestions } \\
\text { du commettant }\end{array}$ & 11 & 17 & 4 & 5 & 37 \\
\hline Matériaux à l'état brut & 7 & 16 & 3 & 2 & 28 \\
\hline Pièces prétravaillées & 3 & 14 & 3 & 2 & 22 \\
\hline $\begin{array}{l}\text { Intervention directe du client } \\
\text { pour le développement du CQ }\end{array}$ & 3 & 10 & 3 & 5 & 21 \\
\hline $\begin{array}{l}\text { Appareillage pour productions } \\
\text { spécifiques }\end{array}$ & 3 & 6 & 2 & 2 & 13 \\
\hline Formation directe par le client & 0 & 5 & 2 & 3 & 10 \\
\hline
\end{tabular}

Source : ODISSEO-DIS, Facoltà di Ingegneria, Naples 
Ces canaux témoignent encore une fois du fait que la qualité modifie le rapport entre les grandes et les petites entreprises. La grande entreprise ne se limite plus à un contrôle de qualité des parties réalisées par la petite entreprise, lorsqu'elles lui sont livrées, mais assiste le sous-traitant au début de la commande et vérifie, à travers des visites de contrôle, si les conditions générales des petites entreprises assurent la réalisation de composants de qualité.

\section{TABLEAU 5}

Le poids des canaux de transmission de la technologie depuis la grande entreprise de haute technologie jusqu'à la petite sous-traitante

(valeurs en pourcentage de chacune des classes)

\begin{tabular}{|c|c|c|c|c|c|}
\hline Canaux de transmission & & Classes & salariés & & Total \\
\hline de la technologie & $1-20$ & $21-50$ & 51-100 & $>\mathbf{1 0 0}$ & \\
\hline Documents écrits & 88,2 & 100,0 & 100,0 & 90,0 & 94,8 \\
\hline $\begin{array}{l}\text { Collaboration de début } \\
\text { de commande }\end{array}$ & 64,7 & 88,0 & 83,3 & 80,0 & 79,3 \\
\hline $\begin{array}{l}\text { Réunions dans les locaux } \\
\text { du commettant }\end{array}$ & 76,5 & 76,0 & 100,0 & 80,0 & 79,3 \\
\hline Visites du client & 58,8 & 80,0 & 83,3 & 80,0 & 74,1 \\
\hline $\begin{array}{l}\text { Suggestions pour l'adoption } \\
\text { du CQ }\end{array}$ & 47,1 & 80,0 & 83,3 & 60,0 & 67,2 \\
\hline $\begin{array}{l}\text { Visites et suggestions } \\
\text { du commettant }\end{array}$ & 64,7 & 68,0 & 66,7 & 50,0 & 63,8 \\
\hline Matériaux à l'état brut & 41,2 & 64,0 & 50,0 & 20,0 & 48,3 \\
\hline Pièces prétravaillées & 17,6 & 56,0 & 50,0 & 20,0 & 37,9 \\
\hline $\begin{array}{l}\text { Intervention directe du client } \\
\text { pour le développement du CQ }\end{array}$ & 17,6 & 40,0 & 50,0 & 50,0 & 36,2 \\
\hline $\begin{array}{l}\text { Appareillage pour productions } \\
\text { spécifiques }\end{array}$ & 17,6 & 24,0 & 33,3 & 20,0 & 22,4 \\
\hline Formation directe par le client & 0,0 & 20,0 & 33,3 & 30,0 & 17,2 \\
\hline
\end{tabular}

Source : ODISSEO-DIS, Facoltà di Ingegneria, Naples 


\section{Influence de la grande entreprise sur le développement quantitatif et qualitatif des petites entreprises}

De la recherche effectuée, il ressort qu'un processus significatif de développement quantitatif et qualitatif des entreprises sous-traitantes étudiées est en cours. Le tableau 6 reporte le nombre de salariés, le chiffre d'affaire (CA) en millions de dollars US, la croissance du chiffre d'affaire, le chiffre d'affaire par salarié en milliers de dollars US et une prévision des investissements pour la période 1990-1995. La croissance du chiffre d'affaire prévue se révèle toujours supérieure ou au moins égale à $10 \%$. En particulier, l'augmentation du chiffre d'affaire oscille entre un minimum de $7 \%$ et un maximum de $30 \%$. De même, le niveau des investissements programmés est extrêmement élevé : à l'exception d'une seule entreprise, la prévision d'investissements se révèle toujours supérieure à $10 \%$ avec des pointes qui arrivent jusqu'à 30 ou $35 \%$. Ceci met en évidence le fait que l'échantillon d'entreprises fait partie d'un univers d'entreprises en phase de forte croissance aussi bien sur le plan de la quantité que sur celui de la qualité.

Tableau 6

Caractéristiques moyennes des petites entreprises, 1990

\begin{tabular}{|c|c|c|c|c|c|}
\hline $\begin{array}{l}\text { Classes } \\
\text { de salariés }\end{array}$ & Salariés & $\begin{array}{c}\text { CA } 90 \\
\text { (millions } \\
\text { de \$US) }\end{array}$ & $\begin{array}{c}\text { Taux de } \\
\text { croissance } \\
\text { du CA } \\
(90-95\end{array}$ & $\begin{array}{c}\text { Inves- } \\
\text { tisse- } \\
\text { ments } \\
90-95 \\
(\% \text { du CA })\end{array}$ & $\begin{array}{c}\text { CA } \\
\text { par salarié } \\
\text { (milliers } \\
\text { de \$US) }\end{array}$ \\
\hline Jusqu'à 20 & 15 & 1,24 & 13 & 22 & 83 \\
\hline De 20 à 50 & 32 & 2,40 & 13 & 15 & 75 \\
\hline De 51 à 100 & 72 & 5,52 & 12 & 10 & 77 \\
\hline Plus de 100 & 263 & 25,85 & 12 & 14 & 98 \\
\hline Toutes classes & 71 & 6,00 & 13 & 17 & 85 \\
\hline
\end{tabular}

Source : ODISSEO-DIS, Facoltà di Ingegneria, Naples

Bien évidemment, ce processus de développement est en partie autonome, et dans ce cas, il est lié aux spécificités de l'entreprise sous-traitante et à la capacité qu'elle a de donner une réponse aux stimulations qu'elle reçoit de l'environnement extérieur. Il est en partie voulu et guidé par les principales entreprises commettantes qui, comme nous l'avons vu, sont intéressées par le développement d'un réseau de petites et moyennes entreprises en mesure de répondre de façon efficace aux standards de qualité toujours plus pressants 
imposés par la compétition oligopolistique des secteurs respectifs. Gérer et orienter la croissance du réseau ainsi que le flux de matériaux et d'informations correspondants pose au commettant des problèmes nouveaux, celui-ci ne pouvant plus confier la gestion de ces rapports au service traditionnel des achats. Les entreprises développent en leur sein de nouvelles structures, fortement orientées vers la gestion de la qualité, qui sont en mesure de régler sur des bases nouvelles le rapport avec les sous-traitants.

La dimension moyenne des entreprises est d'environ 71 salariés avec un chiffre d'affaire moyen de 6 millions de dollars US. Le taux moyen de croissance annuelle est de $13 \%$ alors que le niveau des investissements prévu par les entreprises pour les cinq prochaines années représente environ $17 \%$ du chiffre d'affaire. La valeur moyenne du chiffre d'affaire par salarié est de l'ordre de 85 mille dollars US. L'observation du comportement en fonction du nombre de salariés montre que la croissance n'est pas homogène. La valeur du chiffre d'affaire par salarié est élevée pour les plus petites entreprises (ayant moins de 20 salariés), diminue pour les entreprises ayant entre 51 et 100 salariés et remonte pour les entreprises ayant plus de 100 salariés (98 mille dollars US). Par rapport à cette tendance, on voit que ce sont justement les entreprises de la classe 51-100 salariés qui programment les investissements les plus bas (10\%). Il apparaît donc un parcours de croissance de ces entreprises où les très petites entreprises, qui disposent d'un rapport chiffre d'affaire/salariés élevé, pensent faire de nouveaux investissements dans le but de réaliser un saut de qualité en regard de la technologie. Une fois dépassé le seuil des 21-50 salariés, qui, comme on a pu le voir dans le paragraphe précédent, correspond à la classe pour laquelle les flux de communication avec la grande entreprise de haute technologie sont les plus forts, les investissements ralentissent et une plus grande intégration avec l'entreprise de haute technologie est réalisée. Dans la phase successive, l'entreprise tend à récupérer en efficience en augmentant le rapport chiffre d'affaire/salariés. Ce qui se réalise à travers de forts investissements qui, dans la classe de plus de 100 salariés, sont d'environ $14 \%$.

Ce processus de développement est induit par les entreprises de haute technologie à travers les différents canaux de diffusion de la technologie. Le développement se manifeste à travers une croissance des quatre composantes de la technologie. Même s'il y a une étroite intégration entre ces différentes composantes, nous essaierons d'évaluer la contribution de chacune d'entre elles en utilisant le modèle général proposé et en l'appliquant ensuite au rapport entre grandes et petites entreprises sous-traitantes.

\subsection{Machines}

Un indicateur de la croissance des petites entreprises est sans aucun doute l'important développement du parc de machines (Santarelli, Sterlacchini et 
Quaglia, 1991). Dans le tableau 7, on reporte l'évolution dans le temps du parc de machines traditionnelles et du parc de machines à commandes numériques. Les machines traditionnelles comptaient 933 unités en 1983, 1184 unités en 1988 et on prévoit 1098 unités en 1993. En conclusion, la courbe de diffusion des machines traditionnelles atteint la maturité durant la période 1983-1988 alors qu'elle est en phase de déclin dans la période 1988-1993.

Les machines à commandes numériques $(\mathrm{CN})$ augmentent à un rythme considérable durant la période 1983-1988, alors qu'ensuite le rythme de croissance ralentit et qu'on prévoit 340 machines en 1993 (ce qui représente une augmentation de 128 unités par rapport à 1988). La courbe de diffusion de la technologie des machines à $\mathrm{CN}$ est caractérisée par la phase d'introduction qui se prolonge jusqu'en 1983, par la phase de forte croissance durant la période 1983-1988, et par la phase de maturité pour la période 1988-1993. Les entreprises de dimensions plus réduites présentent une dynamique technologique plus forte : en 1983 et en 1988, $78 \%$ des machines à commandes numériques étaient en activité dans des entreprises comptant moins de 51 salariés. Ceci met en évidence le fait que pour les entreprises les plus petites, le saut de qualité technologique coïncide avec l'achat de machines à commandes numériques. Par contre, dans les entreprises de plus de 50 salariés, la croissance est plus problématique dans la mesure où elle implique davantage l'ensemble des quatre composantes de la technologie. Dans ce cas, l'innovation coïncide avec le processus d'intégration des différentes composantes de l'entreprise plutôt qu'avec l'introduction de machines utilisant l'informatique.

TABleau 7

Évolution du parc de machines (1983-1993)

\begin{tabular}{|c|c|c|c|c|c|c|c|c|c|}
\hline \multirow[t]{2}{*}{$\begin{array}{l}\text { Classes } \\
\text { de salariés }\end{array}$} & \multicolumn{3}{|c|}{$\begin{array}{c}\text { Machines } \\
\text { traditionnelles }\end{array}$} & \multicolumn{3}{|c|}{$\begin{array}{l}\text { Machines } \\
\text { à commandes } \\
\text { numérique }\end{array}$} & \multicolumn{3}{|c|}{$\begin{array}{l}\text { Centres } \\
\text { d'usinage }\end{array}$} \\
\hline & 1983 & 1988 & 1993 & 1983 & 1988 & 1993 & 1983 & 1988 & 1993 \\
\hline Jusqu'à 20 & 174 & 231 & 188 & 9 & 37 & 67 & 0 & 0 & 8 \\
\hline De 20 à 50 & 420 & 508 & 441 & 47 & 118 & 158 & 0 & 27 & 48 \\
\hline De 51 à 100 & 118 & 109 & 95 & 4 & 21 & 45 & 0 & 0 & 0 \\
\hline Plus de 100 & 271 & 336 & 374 & 11 & 37 & 70 & 0 & 9 & 21 \\
\hline Toutes classes & 983 & 1184 & 1098 & 72 & 212 & 340 & 0 & 36 & 77 \\
\hline
\end{tabular}

Source : ODISSEO-DIS, Facoltà di Ingegneria, Naples 
Le tableau 7 montre également l'évolution des centres d'usinage durant la période 1983-1993. En 1983, aucun centre d'usinage n'était actif parmi les 58 entreprises interviewées ; dans la période 1983-1988, il en a été acheté 36 et à la fin de 1993, un total de 77 centres d'usinage seront en fonction. Le cycle de vie de ces technologies est caractérisé par une période d'introduction qui va de 1983 à 1988, alors que la phase de croissance est en cours actuellement. La période 1988-1993 sera caractérisée par le fait que les machines à $\mathrm{CN}$ augmenteront à un rythme inférieur par rapport à la période précédente, c'est-à-dire qu'elles entreront dans la phase de maturité. Les entreprises commettantes s'orienteront vers les centres d'usinage, c'est-à-dire vers des technologies de production plus intégrées et plus flexibles.

Dans le tableau 8, on reporte l'évolution des appareils de mesure traditionnels et automatisés. La diffusion des machines à commandes numériques incite les entreprises à utiliser des appareils de mesure automatisés et à abandonner les appareils de mesure traditionnels : ces derniers atteignent un maximum de 71 unités en 1988 pour ne diminuer qu'à 70 en 1993. Par contre, les appareils automatisés, introduits durant la période 1983-1988, atteindront le nombre de 54 unités en 1993.

TABleau 8

Évolution du parc d'appareils de mesure traditionnels et automatisés $1983-1993$

\begin{tabular}{lcccccc}
\hline & \multicolumn{7}{c}{ Appareils de mesure } \\
Classes de salariés & $\mathbf{1 9 8 3}$ & $\mathbf{1 9 8 8}$ & $\mathbf{1 9 9 3}$ & $\mathbf{1 9 8 3}$ & $\mathbf{1 9 8 8}$ & $\mathbf{1 9 9 3}$ \\
\hline Jusqu'à 20 & 1 & 9 & 8 & 0 & 2 & 9 \\
De 20 à 50 & 18 & 26 & 27 & 2 & 8 & 22 \\
De 51 à 100 & 1 & 3 & 2 & 0 & 1 & 4 \\
Plus de 100 & 14 & 33 & 33 & 0 & 7 & 19 \\
\hline Toutes classes & 34 & 71 & 70 & 2 & 18 & 54 \\
\hline
\end{tabular}

Source : ODISSEO-DIS, Facoltà di Ingegneria, Naples

Le tableau 9 met en évidence l'évolution des contrôleurs logiques programmables. Dans la période 1983-1988, on passe de 5 à 16 unités avec une augmentation de 11 appareils. Pour fin 1993, 7 unités supplémentaires sont prévues. 
TABLEAU 9

Évolution des contrôleurs logiques programmables (1983-1993)

\begin{tabular}{lccc}
\hline Classes de salarlés & \multicolumn{3}{c}{ Contrôleurs loglques programmables } \\
& $\mathbf{1 9 8 3}$ & $\mathbf{1 9 8 8}$ & $\mathbf{1 9 9 3}$ \\
\hline Jusqu'à 20 & 0 & 6 & 8 \\
De 20 à 50 & 0 & 1 & 2 \\
De 51 à 100 & 5 & 8 & 12 \\
Plus de 100 & 0 & 1 & 1 \\
\hline Toutes classes & 5 & 16 & 23 \\
\hline
\end{tabular}

Source : ODISSEO-DIS, Facoltà di Ingegneria, Naples

L'évolution des systèmes de conception et d'interface conceptionproduction est reportée dans le tableau 10. Les systèmes CAO passeront de 7 unités en 1988 à 9 unités en 1993. Le nombre de systèmes FAO restera le même jusqu'en 1993 ( 2 unités). Les systèmes CFAO se développeront fortement dans un proche avenir, passant de 11 unités actuellement à 31 unités en 1993. Il semble évident que les entreprises aient renoncé à acheter des systèmes simples, qui ne résolvent pas les problèmes d'intégration entre les différentes fonctions présentes au sein de l'entreprise et avec l'entreprise commettante, et qu'elles trouvent plus cohérent de s'orienter vers les systèmes intégrés de conception et de production, lesquels permettent une plus grande intégration entre les deux fonctions. De cette façon, l'entreprise peut recevoir directement le projet ou le dessin du commettant, elle peut le modifier et l'adapter aux caractéristiques internes, définir le cycle de production et transmettre directement ce dernier aux machines à commandes numériques ou aux centres d'usinage.

TABLEAu 10

Situation et évolution des systèmes de conception et d'interface conception-production

\begin{tabular}{lcccccc}
\hline Classes de salariés & \multicolumn{2}{c}{ CAO } & \multicolumn{2}{c}{ FAO } & \multicolumn{2}{c}{ CFAO } \\
& $\mathbf{1 9 8 8}$ & $\mathbf{1 9 9 3}$ & $\mathbf{1 9 8 8}$ & $\mathbf{1 9 9 3}$ & $\mathbf{1 9 8 8}$ & 1993 \\
\hline Jusqu'à 20 & 1 & 2 & 3 & 4 & 3 & 10 \\
De 20 à 50 & 4 & 5 & 1 & 2 & 8 & 20 \\
De 51 à 100 & 0 & 0 & 1 & 1 & 0 & 0 \\
Plus de 100 & 2 & 2 & 1 & 1 & 0 & 1 \\
\hline Toutes classes & 7 & 9 & 6 & 8 & 11 & 31 \\
\hline
\end{tabular}

Source : ODISSEO-DIS, Facoltà di Ingegneria, Naples 


\subsection{Règles d'organisation}

La recherche a montré que les projets de croissance des petites entreprises visent aussi bien l'expansion géographique du marché (33 entreprises sur 58), que le développement de nouvelles méthodologies de production afin de mieux satisfaire les nouveaux standards, très stricts, de qualité et de fiabilité des commandes (30 entreprises sur 58). Ces deux facteurs ont de fortes implications sur les règles d'organisation. En particulier, les règles d'organisation à l'intérieur de l'entreprise se modifient (Gerwin, 1985), de même que la façon dont l'entreprise règle et gère ses rapports avec l'environnement extérieur.

Pour une grande partie des entreprises, les problèmes de type logistique sont devenus extrêmement pressants ; 17 entreprises sur 58 prévoient le transfert de leur siège social d'ici cinq ans. Les locaux sont désormais devenus trop petits pour satisfaire la nouvelle croissance, et il devient important de réorganiser l'entreprise afin de tenir compte du saut de qualité, en partie déjà réalisé par les entreprises.

En outre, 16 entreprises sur 58 s'orienteront vers des formes de coopération entre entreprises. Ceci est un aspect qu'il faut souligner, puisque certaines grandes entreprises de haute technologie, non seulement orientent et guident les petites entreprises vers un saut qualitatif, mais favorisent également des formes d'association entre les sous-traitants qui permettent la réalisation conjointe de certaines phases complexes et coûteuses du cycle de production qui, autrement, ne seraient réalisables qu'au sein de grandes entreprises.

Le consortium CTS est l'un des exemples les plus récents. Les entreprises du secteur mécanique de précision qui travaillent sur commande pour Aeritalia, encouragées par celle-ci, ont constitué un consortium pour la réalisation des traitements de surface. L'avantage pour Aeritalia est considérable puisque, actuellement, cette phase du processus de production, qui nécessite des installations très coûteuses, n'est réalisable qu'au sein d'Aeritalia. Les petites entreprises réalisant une pièce ou un composant qui doit subir ce traitement opèrent en trois phases. La première a lieu au sein de l'entreprise sous-traitante, la seconde est réalisée dans les locaux d'Aeritalia, et la troisième, de nouveau au sein de l'entreprise sous-traitante. De cette façon, Aeritalia vit la contradiction d'être devenue le carrefour de phases de transformation qu'elle a depuis longtemps renvoyées vers de petites entreprises. Grâce au consortium CTS, Aeritalia $n$ 'aura plus besoin de faire les traitements de surface : les petites entreprises pourront se servir des installations du consortium CTS pour effectuer cette phase clé de leur cycle de production et obtenir ainsi une plus grande rentabilité de leur commande. 
La croissance des petites entreprises sous-traitantes ne se manifeste pas exclusivement à travers le développement interne. Elle est également le fruit d'un rapport de type nouveau avec le système technologique extérieur, créant un réseau de rapports aussi bien avec les grandes entreprises auxquelles elles offrent leur compétence qu'avec les autres petites entreprises avec lesquelles elles instaurent des rapports de compétition pour l'obtention de commandes, la collaboration pour la réalisation de parties et de composants ou pour échanger des conseils et des informations.

\subsection{Capacités professionnelles}

L'enquête sur le terrain met en évidence que, dans les cinq prochaines années, 17 entreprises sur 58 introduiront de nouvelles machines, et 25 entreprises augmenteront leur capacité de production. Ceci entraînera une pression considérable sur les ressources humaines, puisque les entreprises auront besoin de figures professionnelles d'une expertise professionnelle pouvant gérer ces nouvelles technologies : 31 entreprises sur 58 ont prévu l'embauche de nouveaux salariés dans les prochaines années afin d'adapter les ressources humaines aux nouvelles exigences quantitatives et qualitatives de l'entreprise. Puisque les changements des petites entreprises de l'échantillon sont extrêmement importants et qu'ils concernent toutes les fonctions de l'entreprise, les ressources humaines impliquées dans ces changements sont nombreuses et situées à tous les niveaux de l'entreprise : de l'opérationnel à celui des employés et des cadres moyens jusqu'à celui des cadres supérieurs et des dirigeants.

En ce qui concerne le niveau opérationnel, les petites entreprises vont dans le sens d'une intégration des figures professionnelles déjà existantes à l'intérieur de l'entreprise avec des ressources humaines en mesure d'utiliser sur les nouvelles technologies à commandes numériques. C'est le cas des opérateurs sur machines à commandes numériques et des personnes chargées de l'entretien des nouvelles technologies de production. La personne chargée de faire fonctionner la machine à commandes numériques est un opérateur qui, contrairement au tourneur traditionnel, est en mesure d'opérer sur des machines-outils appartenant à différentes familles et dotées d'unités électroniques de programmation et contrôle. Les connaissances de base de cette profession sont différentes de celles des professions traditionnelles puisque, à côté des connaissances traditionnelles, il faut posséder des éléments d'électronique digitale. La personne chargée de l'entretien doit être en mesure d'effectuer des interventions d'entretien ordinaire et extraordinaire sur des machines, des installations et des systèmes automatisés (programmateurs logiques programmables, machines à commandes numériques, centres d'usinage, ...) en intervenant aussi bien sur les composantes mécaniques que sur les unités électroniques de programmation et de gestion. 
En ce qui concerne le niveau des employés et des cadres moyens, une expertise professionnelle en mesure d'opérer avec des systèmes de conception et d'interface conception/production se développe. C'est le cas de l'opérateur CFAO qui doit effectuer des activités de conception/dessin assisté par ordinateur, analyser l'activité de conception en tenant compte des spécifications générales de conception, des méthodologies et des procédures de l'entreprise. Il doit en outre mettre en place et développer des banques de données pour l'entreprise, qui peuvent servir aussi bien pour les applications CAO que pour les applications FAO. Cette nouvelle figure intègre des connaissances traditionnelles, comme par exemple le dessin technique, la géométrie analytique, la trigonométrie et la conception, avec la connaissance de l'informatique.

En ce qui concerne le niveau des cadres supérieurs, des figures professionnelles se développent dans le domaine de la qualité et de la logistique. Le responsable de la logistique doit être en mesure de gérer le flux de parties et de composants aussi bien à l'intérieur de l'entreprise qu'en ce qui concerne les rapports avec les commettants. La logistique s'occupe du développement et de la réalisation de plans, de programmes et de systèmes capables de garantir le flux matériel tout au long du processus de production, depuis l'approvisionnement jusqu'à la livraison au commettant. Les connaissances de base de cette profession sont l'informatique ainsi que les méthodes et techniques de programmation et de planification. Dans ces entreprises, le responsable de la qualité est indispensable pour respecter les standards imposés par le commettant. Il opère en utilisant des systèmes informatiques, il est en mesure de suivre tout le processus de production et d'élaborer la banque de données relative aux problèmes liés à la qualité.

Pour acquérir ces connaissances professionnelles, la petite entreprise s'adresse à la grande (la formation directe par le commettant est l'un des canaux de transmission de la technologie), recourt à la formation auprès des producteurs de machines à commandes numériques ( 28 cas sur 58 ) et à l'embauche de techniciens ( 20 cas sur 58 ).

\subsection{Documents}

La composante de la technologie que l'on a définie comme documents se modifie rapidement sous l'effet de trois facteurs principaux.

Le premier facteur est représenté par le changement des trois autres composantes de la technologie, c'est-à-dire l'introduction de nouvelles machines et de nouveaux systèmes, les nouvelles règles d'organisation et les nouvelles connaissances professionnelles. Par exemple, l'introduction de machines à CN simultanément à celle de systèmes CFAO modifie radicalement la façon de gérer 
le cycle de production. En effet, la documentation qui concerne un cycle de production spécifique est réalisée par un opérateur au FAO. Cet opérateur utilise un logiciel, gère une banque de données ou une feuille de calcul et envoie directement les informations à la machine à travers un réseau. L'introduction de systèmes CAO et CFAO permet une plus grande intégration entre la conception/ dessin du produit, la conception du processus de production et la phase de production elle-même, puisqu'elle rend le flux d'informations circulaire.

Le second facteur concerne le rapport avec l'environnement extérieur et en particulier les rapports de collaboration avec la grande entreprise et les liens avec les autres petites entreprises. Le système d'information de la petite entreprise s'ouvre et se projette vers la grande entreprise commettante, vers les petites entreprises avec lesquelles elle collabore pour la réalisation d'un produit ou d'une commande spécifique et vers l'environnement extérieur en général. De fait, on a souligné plus haut que les documents écrits (55 cas sur 58), les réunions dans les locaux du commettant ( 46 cas) et les visites du client ( 37 cas) représentent pour la petite entreprise d'importants canaux d'acquisition de la technologie de la grande entreprise de haute technologie. On a également vu que 22 entreprises sur 58 renvoient une partie du cycle de production vers d'autres petites entreprises. L'utilisation de canaux comme les publications spécialisées (41 cas), les rapports avec l'université (16 cas), la participation à des congrès et des séminaires (13 cas), et la participation à des foires et des expositions spécialisées (39 cas) témoigne également des rapports avec l'environnement extérieur.

Le troisième facteur concerne les problèmes de gestion de la qualité. Le respect des standards de qualité imposés par les grandes entreprises de haute technologie nécessitent le développement d'une fonction de la qualité adaptée, laquelle se fonde bien évidemment sur une documentation appropriée et sur le traitement des informations relatives autant au processus de production qu'à l'entreprise toute entière (magasin en entrée et en sortie, cycle de production, etc.). L'importance du contrôle qualité (CQ) est mis en évidence par le fait que toutes les entreprises effectuent le CQ sur les matériaux en entrée, que 40 entreprises ont conçu un manuel de CQ. Dans 41 cas, la grande entreprise fait des suggestions pour l'adoption du CQ et dans 24 cas, le commettant intervient directement pour améliorer et accroître le CQ.

\section{Conclusion}

Dans les années 80, la complexité croissante du produit et la problématique de la qualité ont profondément modifié le rapport entre la grande entreprise de haute technologie et les petites entreprises sous-traitantes. $\AA$ la base des 
nouveaux rapports entre entreprises commettantes et entreprises sous-traitantes, on peut relever quatre points clés mis en évidence par cette recherche :

- Dans les secteurs de haute technologie, la complexité des produits/ processus incite les entreprises à développer un système de relations nouvelles où, à l'intérieur d'une organisation hiérarchique, les petites et les grandes entreprises contribuent, même si c'est avec des rôles différents, à la réalisation du produit.

- La recherche de l'efficience/l'efficacité ne peut plus concerner chacune des entreprises de façon indépendante ; elle doit être effectuée au niveau du système tout entier, et donc également à travers le système de relations entre les entreprises sous-traitantes et les entreprises commettantes.

- La qualité est en train de devenir l'un des « principaux instruments de gestion » et le « langage » de communication entre les entreprises. En particulier, la qualité est en train de prendre un rôle de plus en plus important dans le rapport de collaboration entre les entreprises soustraitantes et les entreprises commettantes.

- Dans les secteurs de haute technologie, les rapports de sous-traitance sont en train de subir de profondes modifications. Ils ne se fondent plus sur la correspondance entre convenance des délais de livraison et prix, mais ils jouent également sur les conditions technologiques, financières et de qualité que présente l'entreprise sous-traitante.

Une première conséquence de ce nouveau rapport de sous-traitance est la stratification hiérarchique du niveau des sous-traitants, à l'intérieur de laquelle il faut identifier les entreprises les plus fiables. Il s'agit d'entreprises ayant un haut degré de dynamisme, prêtes à répondre aux sollicitations qui viennent de différentes directions, qui veulent croître, aussi bien sur le plan quantitatif que sur le plan qualitatif - comme en témoignent leurs programmes de développement - et qui réservent une grande place à l'innovation.

Une deuxième conséquence concerne la sélection de sous-traitants qui deviennent des « partenaires principaux » et qui jouent le rôle d'anneaux de conjonction entre l'entreprise de haute technologie et d'autres sous-traitants. De cette façon, les rapports entre entreprises sous-traitantes augmentent, et il se crée un réseau de relations entre les entreprises de plus petites dimensions. Ceci provoque une réduction du nombre de sous-traitants qui ont un rapport direct avec l'entreprise de haute technologie commettante.

Enfin, il est évident qu'un processus de verticalisation de l'activité de sous-traitance est en cours. L'entreprise de haute technologie utilise les entre- 
prises «partenaires principaux » pour verticaliser le cycle de sous-traitance. $\grave{A}$ son tour, l'entreprise " partenaires principaux » décentralise une partie de ses activités vers des entreprises sous-traitantes de second niveau.

Le changement en cours remet en question l'approche compétitive de Porter qui voit dans les fournisseurs (et donc également dans le sous-traitant) des concurrents pour les entreprises du secteur. Dans cette nouvelle optique, le soustraitant devient un partenaire, puisque la qualité des grandes entreprises dépendra toujours davantage de la qualité des composants réalisés à l'extérieur et donc également par les entreprises sous-traitantes.

La grande entreprise de haute technologie, qui fait réaliser à l'extérieur des parties de plus en plus importantes du cycle de production, développe des formes d'organisation en mesure de gérer la nouvelle dynamique de production et de jouer un rôle nouveau afin d'obtenir l'intégration entre les activités internes et les activités externes : celui de solliciter et de soutenir la croissance des petites entreprises sous-traitantes. Les avantages qu'elle en retire sont évidents : elle évite de rester piégée par les entreprises sous-traitantes peu dynamiques et inadaptées aux nouveaux besoins qualitatifs du marché ; elle ne risque pas d'être abandonnée justement par celles des petites entreprises sous-traitantes qui sont les plus dynamiques et qui aspirent à entrer en compétition sur la qualité plutôt que sur le prix.

La petite entreprise, qui se substitue à la grande entreprise dans des parties importantes du cycle de production, devient un acteur et n'est plus un sujet marginal du processus de production. Ceci lui impose de profonds changements qui visent non seulement l'organisation et les technologies, mais également la culture et la stratégie : elle abandonne l'optique de court terme, qui se révèle inadaptée à sa nouvelle position, et elle se projette vers un processus de croissance quantitative et qualitative. Les avantages sont évidents, puisque le rapport avec le commettant n'est plus régi exclusivement par le prix mais se fonde sur un arbitrage entre prix, aspects technologiques, aspects organisationnels et aspects financiers.

Notre recherche a révélé que les canaux de transmission de la technologie à travers lesquels la grande entreprise de haute technologie sollicite la croissance des petites entreprises sous-traitantes sont : les documents écrits ; les réunions dans les locaux du commettant; le flux de matériaux à l'état brut; la transmission de parties et de composants semi-finis ; les appareillages pour les productions spécifiques ; l'assistance à travers l'activité de formation par le client ; les visites et les suggestions du commettant ; la collaboration de début de commande ; les visites du client ; les suggestions du client pour l'adoption du contrôle qualité et l'intervention directe du client pour accroître la fonction du contrôle qualité. 
Ces onze canaux de transmission de la technologie ont une incidence radicale sur les quatre composantes de la technologie des petites entreprises sous-traitantes, en les projetant vers un développement qui n'est pas seulement quantitatif mais qui est surtout de type qualitatif, comme en témoignent la dynamique des figures professionnelles, le poids considérable des investissements sur le chiffre d'affaire et l'évolution du parc de machines, ainsi que des systèmes de production et d'interface conception/production.

La petite entreprise sous-traitante est en train de s'adapter à la réalité des nouveaux rapports avec la grande entreprise, que ce soit par des programmes d'embauche de nouveau personnel ou à travers la croissance des ressources humaines internes. Les petites entreprises sont en train d'effectuer, en ce qui concerne les figures professionnelles, un saut de qualité considérable qui leur permettra de mieux répondre aux exigences du nouveau rapport de soustraitance.

Les considérations exposées ici concernent bien évidemment les secteurs de haute technologie, mais les premiers résultats de cette recherche, non encore terminée confirment le fait qu'il s'agit de tendances qui concernent déjà d'autres secteurs, et qui les concerneront toujours davantage à l'avenir.

\section{Bibliographie}

ALTERSOHN, C. (1992), De la sous-traitance au partenariat industriel, Paris, L'Harmattan.

AMIN, A. (1989), " A model of the small firm in Italy », in E. Goodman, Small Firm and Industrial District in Italy, London, Routledge.

ANSOFF, I.H. (1969), Business Strategy, Harmondsworth, Penguin Books Ltd.

AUERBACH, P. (1988), Competition : The Economics of Industrial Change, New York, Basil Blackwell.

BELLANDI, G. (1989), La gestione strategica della qualità nei rapporti azienda-mercato, Milano, Giuffré.

BUTERA, F. (1990), Il castello e la rete, Milano, Franco Angeli.

CROSBY, P.B. (1979), Quality Is Free, New York, McGraw-Hill.

DEL MONTE, A. (1991), « Alcuni modelli di interpretazione nei rapporti fra grandi e piccole imprese », Conference on Sub-supply Relationship, Napoli, le $10 \mathrm{mai}$.

Deming, W.E. (1982), Quality, Productivity, and Competitive Position, Cambridge, MIT, Center for Advanced Engineering Study. 
DIOGUARDI, G. (1988), «Venti memorandum per una organizzazione in evoluzione », Workshop internazionale dell'Istituto $R S O$ "L'impresa rete : riconoscerla, progettarla, gestirla », Camogli.

EsPosito, E. et M. RAFFA (1990), «I Rapporti fra piccole e grandi imprese », Sviluppo \& Organizzazione, $\mathrm{n}^{\circ} 122$, p. 79-96.

Esposito, E. et M. RAFFA (1991), «Supply in hi-tech industry : the role of the small businesses », 36th ICBS World Conference, Wien, Jle 24 juin.

Frigenbaum, A.V. (1985), Total Quality Control, New York, McGraw-Hill.

FenNeteau, H. (1990), « Mise en concurrence des fournisseurs ou partenariat? 》, Revue Internationale P.M.E., vol. 3, n² 2, p. 167-191.

FUKUDA, R. (1982), Managerial Engineering, Japanese Standard Association.

Galgano, A. (1990), La Qualità Totale, Milano, Il Sole 24 Ore.

GARDINER, J.P. (1984), « Robust and lean designs with state-of-the-art automotive and aircraft examples », in C. Freeman (éd.), Design, Innovation and Long Cycles in Economic Development, London, Pinter, p. 143-168.

GARVIN, D.A. (1987), "Competing on the eight dimension of quality », Harvard Business Review, n 6, p. 101-109.

GERWIN, D. (1985), «Organizational implication of CAM », Omega, vol. 13, n 5 , p. $443-451$.

Hax, A.C. et N.S. Majluf (1991), The Strategy Concept and Progress : A Pragmatic Approach, New York, Prentice-Hall.

IMRIE, R.F. et J. Morris (1988), Buyer-Supplier Relations in the British Economy : An Empirical Study, Cardiff, University of Wales.

IsHIKAWA, K. (1982), Guide to Quality Control, Tokyo, Asian Productivity Organization.

JURAN, J.M. (1988), Juran on Planning for Quality, Juran Institute.

KAY, N.M. (1984), The Emergent Firm, New York, MacMillan.

LAMMING, R. (1986), « For better or for worse : technical change and buyer - supplier relationships in the U.K. automotive component industry », Conference of Operations Management Association of Great Britain, les 2-3 janvier, University of Warwick.

LASSINI, A. et P. MARITI (1991), « Appunti sui rapporti di collaborazione costruttiva per lo sviluppo innovativo delle imprese di minore dimensione ", $L^{\prime}$ Industria, $\mathrm{n}^{\circ} 2$, p. 249-268.

LAZERSON, M. (1990), Subcontracting as an alternative organisational form to verticalintegrated production, State University of New York, Department of Sociology. 
LORENZONI, G. (1990), L'architettura di sviluppo delle imprese minori, Bologna, il Mulino.

Mattana, G. (1988), Qualità, Affidabilità, Certificazione, Milano, Franco Angeli.

NicoletTI, B. (1987), La gestione della qualità, Milano, Franco Angeli.

PADRONI, G. (1983), « La problematica organizzativa nelle aziende di minore dimensione », dans A.A.V.V., L'organizzazione nell'economia aziendale, Milano, Giuffré.

Petroni, G. et L. POI (1990), «Modelli organizzativi della funzione ricerca \& sviluppo », Sviluppo \& Organizzazione, $\mathrm{n}^{\circ} 119$, p. 51-59.

Porter, M.E. (1985), Competitive Advantage, London, The Free Press.

Pratten, C. (1991), "Piccole imprese ed economie di scala », L'Industria, $\mathrm{n}^{\circ} 2$, p. 269-287.

RoBERTS, E.B. (1987), (éd.) Generating Technological Innovation, Oxford University Press.

ROSENBERG, N. (1982), Inside the Black Box : Technology and Economics, Cambridge University Press.

RosEnberg, N. (1987), "Science and technology in the 20th century 》, Settimane internazionali di storia e studi sull' impresa, Perugia.

SAKO, M. (1987), « Buyer-supplier relations in Britain : a case of japanization? », Conference of Japanization of British Industry, les 17-18 septembre, Cardiff.

SANTARElli, E., A. STERlacchini et F. Quaglia (1991), «Investimenti in macchine e innovazione nelle piccole e medie imprese, Una valutazione del progresso tecnico incorporato $», L^{\prime}$ Industria, $n^{\circ} 2$, p. 289-318.

Sengenberger, W., G. Loveman et M. Piore (1990), The Re-emergence of Small Enterprise : Industrial Restructuring in Industrialized Economies, Geneva, Internationali Labour Organization.

TEECE, D.J. (1986), «Innovazione tecnologica e successo imprenditoriale », L'Industria, $n^{\circ} 4$, p. 605-643.

The Technology Atlas Team (1987), « Components of technology for resources transformation ", Technological Forecasting and Social Change, vol. 32, $\mathrm{n}^{\circ} 1$, p. 19-35.

Twiss, B.C. (1974), Managing Technological Innovation, London, Longman.

VACCA', S. (1989), Scienza e tecnologia nell' economia delle imprese, Milano, Franco Angeli.

VACCA', S. (1989), Scienza e tecnologia nell' economia delle imprese, Milano, Franco Angeli. 